[ORIGINAL ARTICLE

Volume 16 Issue 12021

DOI: 10.21315/aos2021.16.1.5

ARTICLE INFO

Submitted: 9/2/2021

Accepted: 21/4/2021

Online: $25 / 6 / 2021$

\section{The Significance of Knowledge, Instructions, Habits and Denture Hygiene Practice in Relation to the Maintenance of Denture Hygiene at Hospital USM's Dental Clinic}

\author{
Nur Syatirah Mohd Noor, Nor Aidaniza Abdul Muttlib*, Adam Husein \\ School of Dental Sciences, Universiti Sains Malaysia, Health Campus, \\ 16150 Kubang Kerian, Kelantan, Malaysia \\ *Corresponding author: aidaniza@usm.my
}

How to cite this article: Mohd Noor NS, Abdul Muttlib NA, Husein A (2021). The significance of knowledge, instructions, habits and denture hygiene practice in relation to the maintenance of denture hygiene at Hospital USM's dental clinic. Arch Orofac Sci, 16(1): 49-55. https://doi.org/10 $.21315 / \operatorname{aos} 2021.16 .1 .5$

To link to this article: https://doi.org/10.21315/aos2021.16.1.5

\begin{abstract}
Good denture hygiene is crucial for the prevention of various periodontal diseases, dental caries and dental stomatitis, which can give rise to a negative impact on the general health of denture users. A comparison exercise was conducted, to determine the effectiveness of information on denture care, provided by different groups of dental personnel. A self-administered and structured questionnaire was distributed to patients who attended Hospital USM's dental clinics, from 2014 to 2019 based on the attendance register of the clinic. The denture hygiene status of 100 participants was assessed during the interview session. The results derived through the questionnaire revealed that $100 \%$ of the respondents, who received both verbal and written instructions, practiced good dental hygiene. It was observed that the most effective instructions received by the respondents were delivered by the dental specialists. Respondents, who clean their dentures with denture cleaning tablets, soap, or denture cleaning paste, were observed to possess better denture hygiene than those who do not. Also, respondents who visit their dentists once every six months for routine dental examinations boasted a higher percentage of good denture hygiene $(81.8 \%)$. A positive association was perceived, between knowledge and the status of denture hygiene.
\end{abstract}

Keywords: Denture care; denture cleansers; denture hygiene; removable partial denture

\section{INTRODUCTION}

The lack of a proper cleaning routine among removable denture users, can increase oral and systemic infections (Coulthwaite \& Verran, 2007; Sumi et al., 2007). It has been established that the development of dental plaque, is closely associated to oralrelated problems such as halitosis, caries and periodontal diseases, as well as other medical conditions such as bacterial pneumonia, infective endocarditis, chronic obstructive lung diseases, and gastrointestinal infection (Coulthwaite \& Verran, 2007). It has also been reported that the occurrence of denture stomatitis is closely linked to poor denture hygiene (Evren et al., 2011).

A combination of mechanical and chemical cleaning, has proven to be the most effective method to guarantee good denture hygiene (Baba et al., 2018; Papadiochou \& Polyzois, 
2018). It has been reported that continuous wearing of dentures contributed towards increased accumulation of plaque (Bates \& Addy, 1978). As such, the cleaning, and subsequent soaking of dentures in a cleaning solution before sleep, is recommended, to reduce the colonization of candida species (Verhaeghe et al., 2019).

The lack of good oral hygiene can lead to a significant increase in the development of plaque, at the lingual and buccal areas of the dentures (Addy \& Bates, 1979). Therefore, it is crucial that more effective educational and reinforcement methods be introduced, to provide denture wearers with the proper knowledge and instructions, regarding good denture hygiene (Milward et al., 2013). In order to ensure good denture and overall health care, denture-wearing patients should be well-informed on the importance of regularly removing their dentures for cleaning, and the necessity to visit the dentist at least once a year (Coates, 1995).

It has been reported that the verbal delivery of instructions alone, is not very effective, to ensure the patients practice good oral hygiene. In view of this, it is recommended that verbal instructions on the plaque removal technique, to be accompanied with a physical demonstration (Chowdhary \& Chandraker, 2011). Additionally, patients need to be properly advised on their choice of chemical solution used for the cleaning of their dentures. This is important, as certain types of chemical solutions can have a specific effect on denture quality. For instance, it has been reported that the use of a sodium bicarbonate-containing denture cleaner, can have an adverse effect on the attachment system of dentures (Kürkcüoğlu et al., 2016).

Regular denture hygiene care is a necessity, for the maintenance of good general oral health, and the prevention of various periodontal diseases, dental caries and denture stomatitis (Addy \& Bates, 1979; Coulthwaite \& Verran, 2007; Milward et al., 2013). To the best of the authors' knowledge, no studies have been conducted at Hospital Universiti Sains Malaysia (Hospital USM), regarding the correlation between the methods employed for knowledge and instruction dissemination, denture wearing habits and the status of denture hygiene. As such, this study focused on discerning the correlation between knowledge, habits and denture hygiene, as well as identifying the group of dental care providers that could impart appropriate instructions to patients, regarding denture hygiene.

\section{MATERIALS AND METHODS}

This cross-sectional study was carried out at the dental clinics of Hospital USM and patients who patronised the dental clinics for the past five years from 2014 to 2019 were identified through the attendance register of the clinic. The inclusion criteria encompassed denture-wearing patients, with an acceptable capacity for reading and comprehending bahasa Malaysia. Physically and mentally disabled patients were excluded from this exercise. The purposive sampling method was employed for the selection of 100 respondents.

Questionnaire by Milward et al. (2013), about 'Knowledge on Removal Denture Wearers on Denture Hygiene' was adopted for this undertaking. The questionnaire, which was translated into bahasa Malaysia and piloted by ten dental students, was separated into two sections. Section 1 consisted of general information of the patient including gender, education status, type and level of understanding of the instruction given, the person who delivered the instruction, and the denture hygiene practice. Section 2 consisted of the information on the type of denture and denture hygiene status. While Section 1 was to be completed by the respondents, Section 2 was to be completed by the researcher. Prior to the distribution of the questionnaires, the respondents were briefed on the objectives of this investigation. 
The assessment for denture hygiene status was conducted according to the approach employed by Hoad-Reddick \& Grant (1988), which is based on the levels of debris and stain. Dentures were categorised as clean (no soft/hard debris or stain), moderate (soft debris and/or hard debris or stains were present around the gingival margins, and were lingual to the mandibular central incisors, or buccal to the maxillary molars) or poor (soft debris was packed over the tissue surface of the denture, and/or hard debris and stains covered the teeth, flanges, tissue surface, and palate) (Hoad-Reddick \& Grant, 1988). The denture hygiene examination was carried out by a single examiner, and calibration was performed by an experienced prosthodontist.

Data analysis was performed with the utilisation of IBM SPSS version 24 . Descriptive statistics analysis included categorical data (presented as frequency and percentage), and numerical data (presented as mean, standard deviation, median, and in an interquartile range).

\section{RESULTS}

The one hundred participants selected for this investigation ranged in age from 42 to 68 years old, with 55 years old as the mean. The education level of all respondents is presented in Table 1. As shown in Table 2, the majority of respondents portrayed good denture hygiene $(68 \%)$, while the denture hygiene of the rest was revealed to be moderate $(26 \%)$, and poor $(6 \%)$.

The assessment results concerning the association between denture hygiene knowledge, and denture hygiene status, are displayed in Table 3. Out of the 100 respondents, 87 received instructions in verbal form from dental personnel, while 11 received instructions in both verbal and written form. Two of the respondents claimed that they never received any instruction from the dental personnel. Eighty-six respondents declared that they understood the instructions delivered. All 11 respondents, who received instructions in both verbal and written form, portrayed an elevated status of denture hygiene.

Table 1 Educational level of the respondents

\begin{tabular}{lr} 
Education & $\boldsymbol{n}(\%)$ \\
No formal education & $0(0)$ \\
Primary school & $4(4)$ \\
Secondary school & $61(61)$ \\
University & $35(35)$ \\
\hline
\end{tabular}

Table 2 Cleanliness of denture

\begin{tabular}{lr} 
Cleanliness of denture & $\boldsymbol{n}(\%)$ \\
Poor & $6(6)$ \\
Moderate & $26(26)$ \\
Good & $68(68)$ \\
\hline
\end{tabular}

While the majority of respondents, who found the instructions comprehensible, demonstrated good denture hygiene, poor denture hygiene still persisted, in $7 \%$ of respondents in this category. Seventyeight percent of the respondents removed their dentures before sleep at night. All respondents affirmed that they cleaned their dentures daily, while 30 respondents claimed that they did so twice a day. Eighty percent of the respondents who cleaned their dentures twice a day had portrayed good denture hygiene. Twenty-six respondents claimed that they cleaned their dentures thrice a day. Nevertheless, less than good denture hygiene persisted in $30.8 \%$ of respondents in this group.

In terms of denture cleaning, the majority of respondents (30\%) used a toothbrush, while the rest used water $(15 \%)$, toothpaste $(20 \%)$, soap $(2 \%)$, denture cleaning tablets $(13 \%)$, denture cleaning paste $(2 \%)$, and others $(18 \%)$. All respondents who cleaned their denture either with denture cleaning tablets, soap, or denture cleaning paste, enjoy good denture hygiene. Respondents, who visited the dentist once every six months for routine dental examinations, were found to have a higher percentage of good denture 
Table 3 Association between knowledge on denture hygiene and denture hygiene status

\begin{tabular}{|c|c|c|c|}
\hline \multirow{2}{*}{ Determinants } & \multicolumn{3}{|c|}{ Denture hygiene status $n(\%)$} \\
\hline & Poor & Moderate & Good \\
\hline \multicolumn{4}{|c|}{ Method for delivery of instructions/ advice on denture hygiene and care, upon receiving denture } \\
\hline Verbal only & $6(6.9)$ & $26(29.9)$ & $55(63.2)$ \\
\hline Written only & $0(0.0)$ & $0(0.0)$ & $0(0.0)$ \\
\hline Both verbal and written & $0(0.0)$ & $0(0.0)$ & $11(100.0)$ \\
\hline No instruction given & $0(0.0)$ & $0(0.0)$ & $2(100.0)$ \\
\hline \multicolumn{4}{|c|}{ Level of understanding regarding instructions delivered } \\
\hline Low & $0(0.0)$ & $2(16.7)$ & $10(83.3)$ \\
\hline Good & $6(7.0)$ & $24(27.9)$ & $56(65.1)$ \\
\hline Not related & $0(0.0)$ & $0(0.0)$ & $2(100.0)$ \\
\hline \multicolumn{4}{|l|}{ Removal of denture at night before sleep } \\
\hline Yes & $6(7.7)$ & $14(17.9)$ & $58(74.4)$ \\
\hline No & $0(0.0)$ & $12(54.5)$ & $10(45.5)$ \\
\hline \multicolumn{4}{|l|}{ Frequency of daily denture cleaning } \\
\hline Once & $2(9.1)$ & $10(45.5)$ & $10(45.5)$ \\
\hline Twice & $0(0.0)$ & $6(11.0)$ & $24(80.0)$ \\
\hline Thrice & $4(15.4)$ & $4(15.4)$ & $18(69.2)$ \\
\hline More than thrice & $0(0.0)$ & $6(27.3)$ & $16(72.7)$ \\
\hline \multicolumn{4}{|c|}{ Denture cleaning methods employed by participants } \\
\hline Water only & $2(13.3)$ & $4(26.7)$ & $9(60.0)$ \\
\hline Toothpaste & $0(0.0)$ & $8(40.0)$ & $12(60.0)$ \\
\hline Toothbrush & $2(6.7)$ & $8(26.7)$ & $20(66.7)$ \\
\hline Denture cleansing tablets & $0(0.0)$ & $0(0.0)$ & $13(100.0)$ \\
\hline Soap & $0(0.0)$ & $0(0.0)$ & $2(100.0)$ \\
\hline Denture cleaning paste & $0(0.0)$ & $0(0.0)$ & $2(100.0)$ \\
\hline Others (Dishwashing liquid, etc.) & $6(33.3)$ & $10(55.6)$ & $2(11.1)$ \\
\hline \multicolumn{4}{|c|}{ Frequency of visit to dentist for routine examination } \\
\hline Once every six months & $2(9.1)$ & $2(9.1)$ & $18(81.8)$ \\
\hline Once a year & $0(0.0)$ & $4(30.8)$ & $9(69.2)$ \\
\hline Once every two years & $0(0.0)$ & $2(100.0)$ & $0(0.0)$ \\
\hline Never & $4(6.3)$ & $18(28.6)$ & 41 (65.1) \\
\hline
\end{tabular}

hygiene (81.8\%). Although there were 63 respondents who never visited the dentist, good denture hygiene was perceived in $65.1 \%$ from this group.

As for the effectiveness of different groups of dental personnel, in terms of denture hygiene care information delivery, specialists head the list with $84 \%$ of respondents demonstrating good denture hygiene, followed by undergraduate students with $78.6 \%$, postgraduate students with $57.7 \%$, and dental officers with $47.6 \%$ (Table 4 ).

\section{DISCUSSION}

Self-administered questionnaires are frequently employed by researchers (McDonald, 2008). However, the use of 
Table 4 Association between instruction giver and denture hygiene status

\begin{tabular}{lcccc} 
& \multicolumn{3}{c}{ Denture hygiene status: $\boldsymbol{n}$ (\%) } & \multirow{2}{*}{$\boldsymbol{p}$-value } \\
\cline { 2 - 3 } Instruction giver & Poor & Moderate & Good & \\
Specialist & $0(0.0)$ & $4(16.0)$ & $21(84.0)$ & \\
Postgraduate student & $3(11.5)$ & $8(30.8)$ & $15(57.7)$ & 0.039 \\
Dental officer & $3(14.3)$ & $8(38.1)$ & $10(47.6)$ & \\
Undergraduate student & $0(0.0)$ & $6(21.4)$ & $22(78.6)$ & \\
\hline
\end{tabular}

this method comes with the risk of biased responses. This can be attributed to the respondents' inclination towards selfenhancement, or their attempt to maintain positivity (Fiske \& Taylor, 1991). Therefore, caution is paramount when interpreting the results. The major limitation of the present study is the ability of patients to recall the information they had received years ago, which they might forget.

The results from the present study revealed that $78 \%$ of the respondents are aware about the need to remove their dentures before sleep at night. Studies conducted by Mushtaq et al. (2019) and Shigli et al. (2015), delivered almost similar results $(70.67 \%$ and $77.1 \%$, respectively). In terms of denture cleaning frequency, all respondents declared that they clean their prostheses daily. This is in agreement with those achieved by Milward et al. (2013), who reported a positive association between frequent denture cleaning, and good denture hygiene. In an investigation conducted by Apratim et al. (2013), it was revealed that only half of the respondents who cleaned their dentures once daily, were able to maintain their dental hygiene. On the other hand, $80 \%$ of the respondents, who cleaned their prostheses twice daily, were blessed with good denture hygiene. This is consistent with the results attained through our investigations. According to Nevalainen et al. (1997), frequent cleaning alone, does not necessarily promote dental hygiene, if the cleaning method employed is inappropriate. This is verified by the respondents involved in our study, who claim that although they clean their dentures more than once daily, a good status of denture hygiene still remains beyond reach.
Generally, slightly more than $60 \%$ of respondents involved in this study demonstrated good denture hygiene. This result is greater than that derived through an investigation conducted by Milward et al. (2013) which reported good denture hygiene among $39.8 \%$ of respondents. In order to realise an improved denture hygiene status among patients, it is imperative that emphasis be placed on instructions related to proper oral hygiene practices.

All $11 \%$ of respondents, who received both verbal and written instructions from dental personnel, exhibited good denture hygiene. This finding is supported by the results from an earlier study conducted by Johnson et al. (2003). Therefore, in comparison to solely a verbal or written instruction approach, a combination of both appears to be the best way forward. However, further studies involving a more appropriate sample size is essential, for the verification of this finding.

Only $15 \%$ of respondents in this study used commercial denture cleansers. This result is significantly lower than the $41.9 \%$ as reported by Milward et al. (2013) in a similar investigation. This is probably due to the higher cost that comes with the use of commercial dental cleansers, in comparison to other cleaning methods. Other contributing factors, to this situation, could be the lack of awareness and information, regarding the effectiveness of commercial denture cleansers. Considering the fact that all respondents who used dental cleansers exhibited good denture hygiene, more emphasis should be placed on explanations regarding the use of these cleansers, during consultation sessions. 
For $63 \%$ of the respondents, dental appointments were never part of their denture care routine. This result is substantially higher than the $15.4 \%$ as reported by Milward et al. (2013), in a similar exercise. This finding may be attributed to a poor education level, the lack of awareness regarding the importance of dental appointments, or the inadequate instructions received during the denture delivery stage. According to the results from this investigation, specialists are the most effective denture hygiene instruction agents. About $84 \%$ of respondents who received instructions from specialists had good oral hygiene, $16 \%$ had a moderate level of oral hygiene, while none exhibited a poor denture status. The effectiveness of the specialist group, when it comes to the delivery of instructions regarding denture care, may be attributed to a better clinician-patient relationship, the extended time devoted to each patient, and the specialist's greater level of experience in this area.

During this investigation, no differentiations were considered between respondents with complete (full) dentures, and those with partial dentures. As the cleaning of complete and partial dentures may call for different skills, it is our recommendation that separate studies be conducted for each group of denture users (those with partial dentures, and those with complete dentures). This will serve to reveal the different levels of understanding and practices of each group. Furthermore, the type of instructions received by the patients should be verified from dental records in future study. In addition to that, it is the best to provide the patient with a daily chart to record their denture cleaning and wearing habits or verify the data with the partner in future study. It is also proposed that future studies be conducted in this area, involving appropriate sample size, to verify the hypothesis, that a combination of verbal and written instructions, is more effective than solely verbal or written instructions in producing better denture hygiene.

\section{CONCLUSION}

For the most part, the respondents selected for this study had good knowledge, and appropriate routines, with regards to the maintenance of denture hygiene. A positive relationship was perceived, between denture hygiene knowledge, and the status of denture hygiene. The delivery of instructions in a verbal and written combination raised the denture hygiene status for all respondents. Specialists lead the way, when it comes to the effective delivery of denture care instructions to denture wearers.

\section{REFERENCES}

Addy M, Bates JF (1979). Plaque accumulation following the wearing of different types of removable partial dentures. $\mathcal{F}$ Oral Rehabil, 6(2): 111-117. http://doi.org/10 $.1111 /$ j.1365-2842.1979.tb01271.x

Apratim A, Shah SS, Sinha M, Agrawal M, Chhaparia N, Abubakkar A (2013). Denture hygiene habits among elderly patients wearing complete dentures. $\mathcal{f}$ Contemp Dent Pract, 14(6): 1161-1164. https://doi.org/10.5005/jp-journals-10024 $-1468$

Baba Y, Sato Y, Owada G, Minakuchi S (2018). Effectiveness of a combination denturecleaning method versus a mechanical method: comparison of denture cleanliness, patient satisfaction, and oral healthrelated quality of life. $\mathcal{F}$ Prosthodont Res, 62(3): 353-358. https://doi.org/10.1016/ j.jpor.2018.01.005

Bates JF, Addy M (1978). Partial dentures and plaque accumulation. $f$ Dent, 6(4): 285-293. https://doi.org/10.1016/03005712(78)90163-X

Chowdhary R, Chandraker NK (2011). Clinical survey of denture care in denture-wearing edentulous patients of Indian population. Geriatr Gerontol Int, 11(2): 191-195. https:// doi.org/10.1111/j.1447-0594.2010.00666.x 
Coates AJ (1995). Denture adhesives: A review. Aust Prosthodont F, 9: 27-31.

Coulthwaite L, Verran J (2007). Potential pathogenic aspects of denture plaque. $B r \mathcal{F}$ Biomed Sci, 64(4): 180-189. https://doi.org/ 10.1080/09674845.2007.11732784

Evren BA, Uludamar A, Işeri U, Ozkan YK (2011). The association between socioeconomic status, oral hygiene practice, denture stomatitis and oral status in elderly people living different residential homes. Arch Gerontol Geriatr, 53(3): 252-257. https://doi.org/10.1016/j.archger.2010.12 .016

Hoad-Reddick G, Grant AA (1988). Prosthetic status: The formation of a schedule. $\mathcal{F}$ Prosthet Dent, 59(1): 105-110. https://doi .org/10.1016/0022-3913(88)90118-7

Fiske ST, Taylor SE (1991). McGraw-Hill Series in Social Psychology. Social Cognition, 2nd edn. New York: Mcgraw-Hill Book Company.

Johnson A, Sandford J, Tyndall J (2003). Written and verbal information versus verbal information only for patients being discharged from acute hospital settings to home. Cochrane Database Syst Rev, 2003(4): CD003716. https://doi.org/10 $.1002 / 14651858 . C D 003716$

Kürkcüoğlu I, Özkir SE, Köroğlu A, Sahin O, Yilmaz B (2016). Effect of denture cleansing solutions on different retentive attachments. F Prosthet Dent, 115(5): 606610. https://doi.org/10.1016/j.prosdent .2015 .11 .012

McDonald JD (2008). Measuring personality constructs: The advantages and disadvantages of self-reports, informant reports and behavioural assessments. Enquire, 1(1): 75-94.
Milward P, Katechia D, Morgan MZ (2013). Knowledge of removable partial denture wearers on denture hygiene. $\mathrm{Br}$ Dent $\mathcal{F}$, 215(10): E20. https://doi.org/10.1038/sj.bdj .2013 .1095

Mushtaq MA, Altaf J, Sheikh MA, Khan MWU, Shah AA (2019). Assessment of knowledge and practices about denture hygiene among complete denture wearers in Lahore city. I Pak Dent Assoc, 28(4): 187-191. https://doi.org/10.25301/JPDA.284.187

Nevalainen MJ, Närhi TO, Ainamo A (1997). Oral mucosal lesions and oral hygiene habits in the home-living elderly. $\mathcal{f}$ Oral Rehabil, 24(5): 332-337. https://doi.org/10 .1046/j.1365-2842.1997.d01-298.x

Papadiochou S, Polyzois G (2018). Hygiene practices in removable prosthodontics: A systematic review. Int $\mathcal{F}$ Dent Hyg, 16(2): 179-201. https://doi.org/10.1111/idh.12323

Shigli K, Hebbal M, Sajjan S, Agrawal N (2015). The knowledge, attitude and practice of edentulous patients attending a dental institute in India regarding care of their dental prostheses. S Afr Dent F, 70(7): 294299.

Sumi Y, Miura H, Michiwaki Y, Nagaosa S, Nagaya M (2007). Colonization of dental plaque by respiratory pathogens in dependent elderly. Arch Gerontol Geriatr, 44(2): 119-124. https://doi.org/10.1016/ j.archger.2006.04.004

Verhaeghe TV, Wyatt CC, Mostafa NZ (2019). The effect of overnight storage conditions on complete denture colonization by Candida albicans and dimensional stability: A systematic review. $\mathcal{f}$ Prosthet Dent, 124(2): 176-182. https://doi.org/10.1016/ j.prosdent.2019.07.014 